RTW rate varied significantly by cancer type. Of workers who returned to work after their first episode of leave after cancer, more than $50 \%$ continued to work after 5 years in largescaled companies. There was a steep decrease in work continuance rates during the first year after RTW, with considerable differences according to cancer site.

Discussion It is very important for companies (especially smalland medium-sized companies) to establish and improve their RTW support system for employees with cancer.

\section{INVESTING IN WORK ABILITY OF PATIENTS WITH IBD: RESULTS OF A PILOT PROJECT ACTIV84WORK (ACTIVATE FOR WORK)}

${ }^{1} \mathrm{M}-\mathrm{N}$ Schmickler, ${ }^{2} \mathrm{~S}$ Coenen, ${ }^{3} \mathrm{D}$ Staes, ${ }^{3} \mathrm{~F}$ Wieme, ${ }^{4} \mathrm{~T}$ Swinnen, ${ }^{5} \mathrm{X}$ Dekeuleneer, ${ }^{6} \mathrm{~T}$ van Hooland, ${ }^{7} \mathrm{~K}$ van den Cruys, ${ }^{2} \mathrm{~S}$ Vermeire. ${ }^{1}$ Mensura Occupational Health Services, Belgium; ${ }^{2}$ Translational Research Centre for Gastrointestinal Disorders, Catholic University of Leuven, Belgium; ${ }^{3}$ Crohn's and Ulcerative Colitis Association, Belgium; ${ }^{4}$ SD Worx, Global Payroll and HR Service Provider, Belgium; ${ }^{5}$ Proximus, Telecommunications Service Provider, Belgium; ${ }^{6}$ Abbvie, Biopharmaceutical research and development, Belgium; ${ }^{7}$ Novellas Healthcare, International Healthcare Recruitment, Belgium

\subsection{6/oemed-2018-ICOHabstracts. 1549}

Introduction Symptoms of inflammatory bowel disease (IBD) (e.g., diarrhoea, urgency, incontinence, and/or fatigue) often make it difficult to actively participate in the workplace and commute to and from work, especially during flare-up periods. Activ84worK was a pilot project to stimulate professional activity and reduce absenteeism in IBD patients by providing them with more flexible working conditions, including teleworking.

Methods Starting in April 2015, IBD patients were recruited for participation by a patient association and the gastroenterology department at University Hospital Leuven. Interested patients were contacted for screening and follow-up throughout the program. Both employees and their employers were followed over a six-month period. The first and last contacts were face-to-face meetings with employees and employers. Five intermediate contacts with employees were conducted by phone or email. The project was evaluated from three perspectives: benefits of teleworking for the employee, benefits for the employer, and the effect on society by measuring absenteeism.

Results Between April 2015 and October 2016, 71 patients showed interest, 19 were eligible to participate, and 14 completed the program. All patients expressed their enthusiasm for teleworking and other tailored and flexible working conditions. Interviews indicated that removing work-related stress factors such as not having a toilet nearby resulted in employees feeling much more at ease. For most patients, this led to lower absenteeism, higher work ability, and lower costs for employers and society. The project was viewed positively by employees and employers alike. More openness was created between both parties and the taboo on the disease was lifted, which had an overall positive impact on patients' work-life balance.

Discussion This pilot project showed that teleworking and flexible working hours improved professional activity among IBD patients. The results could be used to inspire policy-makers and employers to give maximum support to chronically ill people eager to work.
464 COST-EFFECTIVENESS OF A MULTIMEDIA CAMPAIGN TO IMPROVE COPING WITH NON-SPECIFIC LOW BACK PAIN

${ }^{1}$ Arnela Suman, ${ }^{1}$ Frederieke G Schaafsma*, ${ }^{2}$ Rachelle Buchbinder, ${ }^{3}$ Maurits W van Tulder, ${ }^{1}$ Johannes R Anema. ${ }^{1}$ Dept. of Public and Occupational Health, VU University Medical Centre, Amsterdam Public Health research institute, The Netherlands; ${ }^{2}$ Monash Dept. of Clinical Epidemiology, Monash University, Melbourne, Australia; ${ }^{3}$ Dept. of Health Sciences, VU University Amsterdam, Amsterdam Public Health research institute, Netherlands

\subsection{6/oemed-2018-ICOHabstracts. 1550}

Introduction Specific low back problems are a common and costly problem in Western societies. The vast majority $(88 \%)$ of these costs are caused by indirect costs, including job absenteeism, productivity losses, and disability. To reduce these costs, a multimedia campaign was developed for patients with low back pain. This campaign aimed to improve patient cognitions and knowledge regarding back pain, and stimulated active coping including continuing work.

Methods We recruited patients via general practitioners and physiotherapists, and used a RCT design for evaluation. We compared the multimedia campaign to usual care. The campaign consisted of an interactive website, video films, social media and monthly newsletters. Social media included Facebook, Twitter and a patient forum. During the follow-up period of one year cognitions, knowledge, quality of life, absenteeism, and direct and indirect costs were measured and analysed for all patients.

Results 779 patients with low back pain participated, of which 448 patients in the control group and 331 patients in the intervention group. The average age was 56 years, and most patients were women, with a high level of education and a Dutch nationality. In the control group, 232 participants had a paid job, of which 121 reported a physically demanding job. In the intervention group, 183 participants had a paid job, of which 88 reported a physically demanding job. The campaign had no effect on cognitions. The campaign was cost-effective for quality of life, but not for back pain cognitions. The costs for loss of productivity at work were higher in the control group than intervention group, resulting in a potential saving of $€ 748$ per person.

Discussion This multimedia campaign was not effective in improving cognitions in patients with low back pain, but could lead to a reduction in absenteeism costs. From a societal perspective, the campaign was cost-saving.

\section{COLLABORATIVE FACTORS ASSOCIATED WITH POSITIVE ECONOMIC OUTCOMES IN WORK DISABILITY MANAGEMENT - A MULTIPLE CASE STUDY WITH MIXED METHODS}

T Leino*, I Pehkonen, J Turunen, P Juvonen-Posti. Finnish Institute of Occupational Health, Helsinki, Finland

\subsection{6/oemed-2018-ICOHabstracts. 1551}

Introduction Cost of lost labour input in Finland exceed 24 billion $€$ per year. The aim of the study was to find out the content and processes of work ability management and the factors that make it productive. Information of a 20 financial units from Finnish companies employing 600-11 500 were collected from the period 2008 to 2013. 